\title{
Is Easier the Better in a Feasibility Study of Questionnaires?
}

\author{
Jung Ho Park
}

Department of Internal Medicine, Sungkyunkwan University College of Medicine, Kangbuk Samsung Hospital, Seoul, Korea

\author{
Article: Comparative study of 2 different questionnaires in Japanese patients: the quality of life and utility evaluation sur- \\ vey technology questionnaire (QUEST) versus the frequency scale for the symptoms of gastroesophageal reflux \\ disease questionnaire (FSSG) \\ Nonaka T, Kessoku T, Ogawa $Y$, et al \\ (J Neurogastroenterol Motil 2013;19:54-60)
}

The diagnosis of gastroesophageal reflux disease (GERD) is primarily dependent upon symptoms reported by patients. Therefore, several types of questionnaires have been suggested for the diagnosis of GERD and assessment of treatment satisfaction. However, few of standardized questionnaires can be used in the clinical study restrictively due to the lack of validity, reliability and feasibility. The frequency scale for symptoms of GERD (FSSG) was developed by Kusano et $\mathrm{al}^{1}$ in 2004, based on Japanese studies of GERD. The FSSG is composed of twelve questions with cut off score of 8 , and this method showed sensitivity of $62 \%$ and specificity of $59 \%$. The FSSG is suitable for evaluating frequency of typical reflux symptoms. Quality of life and utility evaluation survey technology (QUEST) was suggested as a method of self-administered questionnaire for the diagnosis of GERD. ${ }^{2}$ The QUEST is comprised of 7 items and reports sensitivity of $70 \%$ and specificity of $46 \%$ at the cut-off score of 4. The QUEST includes more specified questions on the pre- cipitating, exacerbating and relieving factors of GERD. In the previous comparative study of FSSG with QUEST, there was no difference in sensitivity, specificity and accuracy for GERD. ${ }^{3}$ Nonaka et al ${ }^{4}$ reported an interesting comparative study regarding the feasibility between 2 questionnaires, QUEST and FSSG. They measured the time taken to complete the questionnaire and counted the number of patients who asked any questions when they filled in the questionnaire. Completion for the QUEST took much longer than for the FSSG, and questions of QUEST were more complicated than those of FSSG. Based on these results, comparative study concluded the FSSG was a more convenient method for clinical use than the QUEST. Results of this study could demonstrate that simplicity is an important feature of a questionnaire and it should be considered critically in future studies. Nevertheless, I would like to make comments on this study. First, since time and number of patients asking questions are indirect parameters of feasibility, there are so many confound-

Received: November 13, 2012 Revised: None Accepted: November 15, 2012

(c) This is an Open Access article distributed under the terms of the Creative Commons Attribution Non-Commercial License (http://creativecommons. org/licenses/by-nc/3.0) which permits unrestricted non-commercial use, distribution, and reproduction in any medium, provided the original work is properly cited.

*Correspondence: Jung Ho Park, MD

Department of Medicine, Sungkyunkwan University School of Medicine, Kangbuk Samsung Hospital, 29, Saemunan-ro, Jongno-gu, Seoul 110-746, Korea

Financial support: None.

Tel: +82-2-2001-2059, Fax: +82-2-2001-2485, E-mail: pjho3@hotmail.com

Conflicts of interest: None. 
ing factors such as educational status of subjects, the language of original questionnaire (translation into Japanese might be responsible for large number of characters and difficulty in understanding), introverted personality (do not want to ask questions) and so on. Therefore, it would be better to ask more direct questions, such as "which one do you like better" and "which questionnaire was easier to understand." Second, 2 questionnaires have quite different questions. QUEST focuses on influencing factors in GERD and FSSG emphasizes on symptom frequency. Therefore, although feasibility is important, considerations on types of questions are needed when we choose a questionnaire.

\section{References}

1. Kusano M, Shimoyama Y, Sugimoto S, et al. Development and evalu- ation of FSSG: frequency scale for the symptoms of GERD. J Gastroenterol 2004;39:888-891.

2. Carlsson R, Dent J, Bolling-Sternevald E, et al. The usefulness of a structured questionnaire in the assessment of symptomatic gastroesophageal reflux disease. Scan J Gastroenterol 1998;33:1023-1029.

3. Danjo A, Yamaguchi K, Fujimoto K, et al. Comparison of endoscopic findings with symptom assessment systems (FSSG and QUEST) for gastroesophageal reflux disease in Japanese centres. J Gastroenterol Hepatol 2009;24:633-638.

4. Nonaka T, Kessoku T, Ogawa Y, et al. Comparative study of 2 different questionnaires in Japanese patients: the quality of life and utility evaluation survey technology questionnaire (QUEST) versus the frequency scale for the symptoms of gastroesophageal reflux disease questionnaire (FSSG). J Neurogastroenterol Motil 2013;19:54-60.

5. Velanovich V. Comparison of generic (SF-36) vs. disease-specific (GERD-HRQL), quality-of-life scales for gastroesophageal reflux disease. J Gastrointest Surg 1998;2:141-145. 